\title{
P03-113
}

\section{A PRELIMINARY STUDY OF P200 IN FIRST-EPISODE PATIENTS, THEIR RELATIVES AND HEALTHY CONTROLS: SEARCH OF NEW ENDOPHENOTYPES}

C. Silveira' ${ }^{1}$, F. Santos ${ }^{2}$, F. Barbosa ${ }^{2}$, A. Pedro ${ }^{2}$, A. Palha' ${ }^{1}$, J. Marques-Teixeira ${ }^{2}$

${ }^{1}$ Serviço de Psiquiatria, Faculdade de Medicina da Universidade do Porto, ${ }^{2}$ Laboratório de Neuropsicofisiologia, Faculdade de Psicologia e de Ciências da Educação da Universidade do Porto, Porto, Portugal

\section{Background/}

Objective: Despite the well established genetic basis of schizophrenia, the relationship between genes and the disorder itself is still elusive. Individual endophenotypes, which reduce the complexity of genetic analyses, allow statistical approaches with quantitative trait methodologies. P200 abnormalities of event-related potentials have been reported in schizophrenia with conflicting results. The present study aims to characterize the P200 in first-episode patients and to compare it with that of first-degree relatives and controls.

Methods: ERPs were recorded at 19 sites with an auditory oddball for 21 first-episode patients with schizophrenia (mean age=25.14; $S D=6.20$ ), 41 of their first degree relatives (mean age=47.65; $S D=15.53$ ) and 19 healthy controls (mean age=26.32; $\mathrm{SD}=7.16$ ). Potentials were averaged for frequent stimuli and P200 amplitude and latency measures were obtained.

Results: Analysis of midline electrodes revealed significant group effects for $P 200$ peak amplitudes $(F(2,78)=3.315, p=.042)$, but not for peak latencies. Post-hoc analyses revealed that patients with schizophrenia present significantly lower P200 amplitudes $(M=2.466 ; S D=1.564)$ than controls $(M=5.037 ; S D=2.500)$ at $P z(T(38)=3.851, p=.003)$. No other significant differences were found.

Conclusion: The results obtained do not straight-forwardly support the P200 peak amplitude nor peak latency as an endophenotype of schizophrenia. However, the trends of our results may suggest that the P200 amplitudes of relatives may present intermediate values between healthy controls (with higher amplitudes) and patients (with lower amplitudes). Further statistical analyses will be required in order to disentangle the effects of possible confounding variables. 